\title{
Inégalités sociales, de santé du constat à l'action - Intérêt de la mise en place d'un accompagnement personnalisé pour la réduction des inégalités sociales en cancérologie
}

\author{
Social inequalities in health from observational studies to intervention: \\ Can the patient navigator reduce social inequalities in cancer patients? \\ O. Dejardin ${ }^{\text {a,* }}$, C. Berchi ${ }^{\text {a }}$, A. Mignon ${ }^{\text {b }}$, C. Pornet ${ }^{\text {a }}$, E. Guillaume ${ }^{\text {a }}$, L. Guittet ${ }^{\text {a }}$ \\ , V. Bouvier ${ }^{\mathrm{a}}$, M. Sailly ${ }^{\mathrm{b}}$, A. Salinas ${ }^{\mathrm{d}}$, V. Christophe ${ }^{\mathrm{b}, \mathrm{c}}$, G. Launoy ${ }^{\mathrm{a}}$ \\ ${ }^{a}$ ERI3 Inserm «cancers et populations », faculté de médecine de Caen, CHU de Caen, avenue de la Côte-de-Nacre, 14032 Caen cedex, France \\ ${ }^{\mathrm{b}}$ Unite' de recherche en sciences cognitives et affectives, URECA EA 1059, universite'Lille-Nord-de-France, universite' de Lille, \\ rue du Barreau, 59653 Villeneuve d'Ascq cedex, France \\ ${ }^{\mathrm{c}}$ MESHS USR CNRS 3185, 59000 Lille, France \\ ${ }^{\mathrm{d}}$ CERReV EA 3918, centre d'études et de recherches sur les risques et les vulnérabilités, université de Caen/Basse Normandie, \\ esplanade de la Paix, 14032 Caen cedex, France \\ Reçu le 22 mars 2010 ; accepté le 14 octobre 2010 \\ Disponible sur Internet le 21 janvier 2011
}

\begin{abstract}
The impact of social factors on healthcare inequality is well-recognized in many industrialized countries and involves a wide range of pathological conditions (cardiovascular disease, cancer, etc.). In general, the poorest indicators of health are observed in socially disadvantaged populations. Beyond this observation is the question of actions taken to prevent the formation of social inequality in healthcare. The purpose of this work was to evaluate the potential contribution of an intervention tool called the "patient navigator", used in English-speaking countries and to determine its feasibility in France.
\end{abstract}

\# 2010 Elsevier Masson SAS. All rights reserved.

Keywords: Social inequalities; Cancer; Colorectal cancer; Intervention; Patient navigator

Résumé

Des inégalités sociales de santé ont été rapportées dans l'ensemble des pays industrialisés et pour un très grand nombre de pathologies (maladies cardiovasculaires, cancers, etc...). Les patients issus des milieux les plus défavorisés présentent généralement les plus mauvais indicateurs de santé. Au-delà du constat se pose dès lors la question des moyens d'action permettant de lutter efficacement contre la formation des inégalités sociales de santé. L'objectif de cet article est d'étudier l'intérêt potentiel d'une méthode de lutte contre les inégalités sociales en cancérologie, couramment dénommée «patient navigator » dans les pays anglosaxons (accompagnateur d'un patient en français) et d'évaluer la faisabilité de sa mise en place en France.

\# 2010 Elsevier Masson SAS. Tous droits réservés.

Mots clés : Inégalités sociales ; Cancer ; Cancer colorectal ; Dépistage ; Accompagnateur ; Intervention ; Patient navigator

\section{Introduction}

Les inégalités sociales sont présentes dans de nombreuses composantes de la société : la justice, l'éducation, le monde du travail, et dans un domaine où elles sont régulièrement mises en

* Auteur correspondant.

Adresse e-mail : Olivier.dejardin@unicaen.fr (O. Dejardin) avant, la santé. Des inégalités sociales de santé ont été rapportées dans l'ensemble des pays industrialisés et pour un 
très grand nombre de pathologies (maladies cardiovasculaires, cancers, etc...) [1]. Les patients issus des milieux les plus défavorisés présentent généralement les plus mauvais indicateurs de santé. La position de la France, pourtant pourvue de l'un des meilleurs systèmes de soins au monde, est bien peu enviable, comme le montrent ces deux chiffres : d'une part, le gradient de mortalite' entre la zone d'emploi du nord du pays et la zone d'emploi du sud varie de plus de dix ans, d'autre part, l'espérance de vie à 35 ans d'un ouvrier français est inférieure de 6,5 années à celle d'un cadre [2]. Grâce à l'utilisation de niveaux géographiques plus discriminants qu'une simple comparaison Nord-Sud, des inégalités géographiques ont été mises en évidence, par exemple pour l'obésité infantile ou pour le tabagisme [3]. Néanmoins, ces approches ne permettent pas de s'affranchir du biais écologique, ce qui limite leur intérêt.

Concernant les pathologies cancéreuses, un célèbre article publié en 1997 par Mackenbach et al. [4] soulignait que la France était le pays où les inégalités de mortalité par cancer étaient les plus fortes en Europe de l'Ouest. Néanmoins, dès lors que l'on cherche à identifier les mécanismes à l'origine de ces inégalités sociales, l'indicateur « mortalité » montre rapidement ses limites. En effet, les inégalités de mortalité sont la résultante des inégalités dans l'incidence des inégalités dans le pronostic des cancers. Or, les facteurs déterminant les inégalités d'incidence et de pronostic ne sont pas du même ordre. Si l'organisation du système de soins intervient peu dans les inégalités d'incidence, qui relèvent principalement de l'exposition environnementale et professionnelle et du mode de vie, son rôle peut être majeur dans les inégalités de survie $[5,6]$.

Malgre' le retard accumule' en France dans le domaine de la recherche sur les inégalités sociales et géographiques de santé [7], les études visant à identifier les facteurs à l'origine de ces disparités se sont multipliées au cours des dernières années [1]. Le même constat que chez nos voisins européens a pu être mis en évidence $[8,9]$ et les études menées à ce sujet mettent en exergue l'importance de l'accessibilité géographique et sociale aux centres de soins spécialisés comme facteur explicatif de ces disparités [10,11]. Les mécanismes potentiels à l'origine de cette «perte de chance socialement déterminée » sont multiples

et divers tout au long du processus de prise en charge des cancers. Il peut s'agir d'un moindre accès au dépistage et à la médecine de premier recours, d'une ignorance des symptômes révélateurs de la maladie, d'une utilisation différente des filières de soins, d'une moindre adhésion au traitement ou au suivi. À chacune de ces étapes, plusieurs déterminants interagissent, d'ordre individuel ou collectif, qui s'organisent dans des modèles explicatifs « comportementaux » ou «structurels ».

Dans cette perspective, au même titre que le cancer du sein, le cancer colorectal représente un bon modèle pour l'étude des mécanismes de formation des inégalités sociales, en particulier parce qu'il est fréquent (plus de 37000 nouveaux cas en 2005) [12], que son pronostic est étroitement lié à la précocité de sa découverte, et qu'il existe une technique valide pour le dépister (recherche de sang occulte dans les selles). Le dépistage des cancers colorectaux par le test Hemocult II $^{\mathbf{1}}$ a été généralisé à l'ensemble de la France en 2008. La Haute Autorite' de sante' envisage désormais la substitution de ce test au gaïac par un test immunologique plus performant. L'étape du dépistage est certainement fondamentale dans la construction des inégalités sociales de survie des patients atteints de cancer colorectal. La faible participation au dépistage est, de fait, étroitement liée à un faible niveau socioéconomique individuel, à un faible niveau d'études et de revenus [13,14]. Une étude récente montre que dans les unités géographiques les plus défavorisées, la participation au dépistage du cancer colorectal est diminuée d'environ un tiers (32\%) par rapport aux zones favorisées [15].

À côté du dépistage, des études récentes soulignent l'importance de l'accès aux traitements initiaux et adjuvants dans la formation des inégalités sociales. Ainsi, l'accès à la chimiothérapie adjuvante explique une part importante des inégalités sociales constatées dans la survie des patients atteints de cancer du colon [8]. Lorsque les déterminants sociaux du traitement sont effacés, par exemple dans un essai randomisé où l'ensemble des patients rec oivent un traitement strictement comparable, il n'y a alors plus d'influence des caractéristiques socioéconomiques du patient sur sa survie [16]. Ces études nous conduisent à penser que l'amélioration de l'accès au dépistage et aux traitements pour les personnes les plus défavorisées constitue un moyen d'action potentiel de réduction des inégalités sociales pour les patients atteints d'un cancer.

Au-delà du constat se pose, dès lors, la question des moyens d'action permettant de lutter efficacement contre la formation des inégalités sociales de santé. Un rapport récent de l'Organisation mondiale de la sante' (OMS) fixe l'objectif

«Combler le fossé en une génération-instaurer l'équité en agissant sur les déterminants sociaux de la santé » (www.who.int/social_determinants). Comme souligne' dans ce rapport, la réduction des inégalités sociales de santé est une problématique intersectorielle qui nécessite la mobilisation de l'ensemble des composantes de la société. Ainsi, comme le montre l'abondante littérature sur les déterminants contextuels de la santé [17,18], l'amélioration des conditions de vie des habitants d'une zone géographique, qui échappe pour une grande part au strict champ de la médecine et de la santé (augmentation des revenus, amélioration de l'habitat...), se traduit par une amélioration de leur santé. Néanmoins, la lutte contre les inégalités sociales de santé passe également par des interventions spécifiques en santé tournées vers les populations défavorisées. Malheureusement, dans ce domaine, l'évaluation des actions est, surtout en France, extrêmement rare et les décideurs ne disposent que de peu d'éléments concernant les moyens d'une action raisonnée.

L'objectif de cet article est d'étudier l'intérêt potentiel d'une méthode de lutte contre les inégalités sociales en cancérologie, couramment dénommée «patient navigator » dans les pays anglosaxons (accompagnateur d'un patient en francsais), et d'évaluer la faisabilité de sa mise en place en France.

\section{Présentation des programmes d'accompagnement}

L'objectif des programmes d'accompagnement des patients est de réduire l'influence des déterminants sociaux de la santé 
en levant l'ensemble des barrières faisant obstacle à une prise en charge optimale des populations défavorisées tout au long de la filière de soins. Ces programmes, quasi-exclusivement entrepris en Amérique du Nord, ont principalement été développés sur la question de l'accès au traitement après un dépistage laissant présager un cancer. Si la majorité des projets d'intervention ciblent en priorité des populations défavorisées, des programmes plus vastes peuvent également concerner une collectivité particulière (typiquement une «Health Maintenance Organization » [HMO], organisme qui intègre la fonction de financeur [assureur] et de producteur de soins).

Le premier programme d'accompagnement qui a été conduit a concerne' un quartier de New York fortement affecte' par la pauvreté. Partant du constat d'une sur-mortalité par cancer du sein dans le quartier de Harlem (New York, États-Unis) au début des années 1990, ce programme a été mis en place par le docteur Freeman afin de lutter contre les disparités subies par les populations défavorisées ayant une suspicion de cancer du sein, du côlon, du col de l'utérus et de la prostate [19,20]. Les trois principaux types de barrières rencontrés par ces populations étaient [21] :

- les barrières financières : non éligibilité au programme de gratuite' des soins Medicare et Medicaid, perte d'emploi ayant entra ine' la perte de l'assurance sante' ;

- les barrières logistiques : problème d'accessibilité physique aux soins ;

- les barrières socioculturelles : problème de compréhension du langage, manque de soutien social.

Les objectifs principaux de cette intervention étaient d'augmenter la participation des populations défavorisées au dépistage des cancers, de fournir un accompagnement aux patients atteints d'un cancer ou présentant des signes anormaux suite à un dépistage et enfin d'inciter les initiatives locales d'éducation vis-à-vis du dépistage. Ce projet a été conduit en trois phases consécutives. Durant les six premiers mois, l'accompagnateur a rencontre' les patients atteints de cancer de l'hôpital de Harlem afin de se familiariser avec leurs problèmes. Durant l'année suivante, il a proposé l'intervention aux patients présentant des signes anormaux suite à un dépistage. Devant la difficulte' d'accompagner deux populations dont les préoccupations étaient très différentes (patients atteints d'un cancer et patients ayant une suspicion de cancer), il a été décidé de s'occuper en priorite' des patients ayant une suspicion de cancer. Enfin, pour les dix mois restant, un second accompagnateur parlant espagnol a été recruté. L'évaluation de cette première étude d'accompagnement a montré que l'intervention avait entrâ ine' une augmentation, non significative probablement en raison d'un effectif relativement faible $(n=1136)$, du nombre de biopsies effectuées dans le groupe ayant reçu l'intervention par rapport au groupe témoin $(85,7 \%$ des patients ayant reçu une biopsie dans le groupe accompagne' contre 56,5\% dans le groupe non accompagné). Néanmoins, le délai de réalisation de la biopsie s'est trouve' diminue' dans le groupe intervention $(71,4 \%$ des patients ont effectue' une biopsie en moins de quatre semaines contre $38,5 \%$ dans le groupe non accompagne' $[p<0,05])$.
Depuis cette étude novatrice, plusieurs dizaines d'interventions mettant à la disposition des plus défavorisés des accompagnateurs se sont développées en Amérique du Nord (États-Unis et Canada). Ces projets d'intervention font aujourd'hui l'objet d'un vaste plan de recherche organise' parle National Cancer Institute (http://www.cancer.gov/cancertopics/ factsheet/nci/patientnavigator). Ce programme de recherche vise à évaluer l'efficacité des programmes d'accompagnement à travers une large étude comprenant neuf sites et quatre localisations cancéreuses. Les critères d'efficacité principaux sont : le délai d'accès au diagnostic, le délai d'accès à un traitement, l'évaluation de la qualité de vie des patients et, enfin, le rapport coût-efficacité du programme. Les critères de jugement secondaires sont : le taux de thérapies complètes, la qualite' des soins, l'activite' des accompagnateurs [22].

Ce programme de recherche est le premier àce jour à proposer une évaluation économique précise. L'accompagnement du dépistage bénéficiant d'un financement socialisé, son efficience doit être démontrée avant d'être généralisé. Les quelques auteurs évoquant la question économique font état d'un coût élevé lié à la mobilisation intensive du facteur travail. Le coût de l'action dépend essentiellement du niveau de qualification de l'accompagnateur. Or, il existe une grande variété de profils professionnels en charge de l'accompagnement (profane, infirmière, assistante sociale, éducateur, assistant de recherche, ou personne ayant survécu au cancer). Les stratégies de réduction des coûts de personnel consistent à recourir à des accompagnateurs moins qualifiés ou à réduire leur temps de formation. Les interventions menées dans le cadre de Medicare et Medicaid envisagent ainsi de remplacer les infirmiers par des travailleurs sociaux. Les expériences passées suggèrent pourtant que cette stratégie est, certes, moins coûteuse mais aussi moins efficace [23].

Il demeure particulièrement difficile de quantifier exactement le nombre de ces programmes d'accompagnement en l'absence, d'une part, d'un cadre formel définissant l'action des accompagnateurs et, d'autre part, d'une banque de données des actions déjà mises en place.

\section{Rôle des accompagnateurs}

Le rôle de l'accompagnateur est d'entreprendre l'ensemble des actions possibles permettant de lever les barrières limitant ou retardant la réception d'un acte médical. Le rôle «pro-actif » de l'accompagnateur est à souligner ici. Son rôle est non seulement d'identifier les barrières d'accès aux soins mais également, dans la mesure du possible, de les anticiper. Sans être exhaustives en raison du caractère individualisé de leur action [21], les modalités d'actions peuvent varier du simple appel téléphonique permettant de mettre en valeur l'intérêt d'un acte de dépistage, à la prise de rendez-vous chez le médecin, à l'organisation de la garde des enfants pendant les soins, à l'accompagnement physique du patient àla consultation, àla possibilité de recourir à un interprète afin d'améliorer la compréhension ou encore au soutien psychologique des patients pendant les soins. En dehors d'un effort d'information, il est à préciser que l'accompagnateur n'effectue, en aucun cas, 
le travail à la place du médecin ou plus généralement de l'ensemble des soignants.

Les accompagnateurs étant à l'interface entre les soignants et les travailleurs sociaux, la formation prérequise est très diverse selon les protocoles d'études. Les accompagnateurs sont issus, selon les études, essentiellement de deux corps de métiers, des infirmières et des assistantes sociales. Dans le contexte américain où les accompagnateurs s'adressent principalement à des communautés ethniques ciblées, ces derniers sont majoritairement recrutés au sein des communautés dont ils vont avoir la charge (Native sisters).

Bien que des travailleurs sociaux soient déjà présents dans les structures de soins, les accompagnateurs se distinguent de ces derniers par le fait que leurs actions sont ciblées sur une population particulière (généralement défavorisée), et qu'ils peuvent être amenés à suivre le patient tout au long de la filière de soins (du dépistage à l'arrêt des traitements). Les accompagnateurs doivent travailler en synergie avec les différents travailleurs sociaux présents dans l'ensemble des structures de soins que va fréquenter le patient au cours de sa thérapie afin d'assurer la meilleure prise en charge possible.

\subsection{Analyse des déterminants psychosociaux de l'influence de l'accompagnateur}

Pour le patient, l'intérêt de l'intervention de l'accompagnateur sur le dépistage réside surtout dans son orientation individualisée, centrée sur ses besoins et ses caractéristiques. Cette orientation individualisée n'est pas sans conséquence psychologique sur son vécu émotionnel et ses croyances en matière de dépistage, ainsi que sur son degré d'adhésion au dépistage et au traitement. En effet, l'accompagnateur peut remplir plusieurs rôles complémentaires, permettant de lever différentes barrières. Il constitue un support social pouvant prendre trois formes non exclusives - émotionnelle, informationnelle et instrumentale - chacune étant plus ou moins mobilisée selon les cas [24]. En ce qui concerne le versant émotionnel, l'accompagnateur peut établir une relation d'empathie et de confiance, permettant au patient d'évoquer d'éventuelles craintes (voire son angoisse ou son stress) face à la procédure de dépistage et au diagnostic qui en découle. Ces craintes constituent des facteurs inhibiteurs que l'accompagnateur peut lever directement avec le patient [25]. Lever les barrières émotionnelles permet également de conduire les patients à prendre des décisions concernant la réalisation du dépistage fondées davantage sur des critères rationnels que sur des critères émotionnels. L'accompagnateur peut aussi avoir pour fonction essentielle de conseiller, d'informer le patient sur la nature des démarches et la manière de les mener, ainsi que sur le cancer lui-même et l'intérêt du dépistage précoce. De ce fait, les croyances « profanes » qui constituent un frein au dépistage peuvent être dépassées pour laisser place à une connaissance plus réaliste et mobilisatrice. En augmentant leur degré de connaissance, les individus peuvent devenir plus favorables au dépistage. Le travail informationnel de l'accompagnateur facilite une prise de décision plus «éclairée ». Enfin, si l'individu décide d'entreprendre un dépistage, l'accompagnateur peut apporter une aide pour la mise en œuvre des actions nécessaires (aide à la planification et àla prise de rendez-vous, par exemple) [26]. Audelà de trouver des solutions aux contraintes rencontrées par le patient pour réaliser ces actions (par exemple, garde des enfants pendant les soins ou les examens) l'aide de l'accompagnateur permet, àun niveau plus psychologique, de renforcer le lien entre les intentions de l'individu de réaliser certaines actions et la réalisation effective. L'individu peut ainsi se sentir davantage engagé dans sa propre démarche, ce qui peut contribuer à augmenter son adhésion au dépistage et au traitement éventuel.

\section{Principaux résultats concernant l'efficacité des accompagnateurs}

\section{Influence des programmes d'accompagnement sur la participation des patients aux campagnes de dépistage}

La participation au dépistage des cancers a souvent été rapportée comme socialement stratifiée [27]. L'une des actions les plus fréquentes a été la mise en place d'un programme d'accompagnateur pour améliorer l'accès aux coloscopies (une des stratégies recommandée pour le dépistage des cancers colorectaux aux États-Unis) pour les populations défavorisées. Ainsi, une étude a été conduite au sein du Lincoln Medical Center, situé dans l'un des quartiers les plus défavorisés de New York [28]. L'intervention consistait àpermettre aux populations défavorisées de contacter deux accompagnateurs situés dans l'hôpital et chargés de faciliter la prise de rendez-vous pour la réalisation de la coloscopie. Cette intervention, conjointe à la mise en place d'un circuit de consultation plus rapide, a permis la chute importante du nombre de rendez-vous annulés et, en conséquence, une hausse significative du nombre de coloscopies réalisées chez les femmes et les bénéficiaires de l'assurance sociale Medicaid (le nombre moyen de coloscopie réalisée par mois étant passé respectivement de 37,5 à 76,0 pour les femmes et de 17,0 à 48,4 pour les patients relevant de Medicaid).

Un essai contrôlé randomisé conduit sur une population défavorisée du Massachusetts a été réalisé dans le but d'augmenter la participation au dépistage des cancers colorectaux de 409 patients [29]. Cinq travailleurs sociaux, possédant tous un diplôme du supérieur et la maîtrise d'une langue étrangère, ont été recrutés en qualité d'accompagnateur. Les accompagnateurs recevaient une formation de six heures sur le dépistage des cancers colorectaux. L'intervention consistait en l'envoi d'une lettre d'information, puis d'un appel téléphonique dans une langue maîtrisée par le patient. Lors de ce contact téléphonique, l'accompagnateur devait conjointement améliorer les connaissances des sujets sur le cancer colorectal et identifier les barrières potentielles au dépistage. Enfin, l'accompagnateur était chargé d'aider le sujet àprendre rendez-vous, de rappeler l'imminence de ce rendezvous, d'informer le sujet sur les modalités de préparation à l'examen et d'organiser le transport. Les auteurs ont montre' une augmentation significative de la participation au dépistage du cancer colorectal $(27,4 \%$ dans le bras intervention contre 11,9 $\%$ dans le bras témoin). L'intervention s'est révélée plus 
efficace pour les sujets âgés, les femmes, les sujets dépourvus d'une assurance privée et pour les sujets n'appartenant pas à la communauté latino-américaine. Enfin, elle était plus efficace pour les sujets parlant anglais que pour les sujets dont la langue anglaise n'était pas la langue maternelle. Les auteurs expliquent ce phénomène par le taux élevé de sujets ayant reçu un dépistage dans le groupe témoin, et dont la langue anglaise n'était pas la langue maternelle témoin d'une probable contamination entre les groupes.

Une étude conduite à New York a concerné la coloscopie de dépistage du cancer du côlon [30]. L'intervention, conduite par une professionnelle bilingue de l'éducation à la santé, consistait en un appel téléphonique suivi d'instruction par courriel sur la préparation à la coloscopie, et enfin de deux rappels respectivement àdeux semaines et à trois jours de l'examen. Les patients ayant reçu un accompagnement étaient près de $66 \%$ àavoir effectue' cette coloscopie (en l'absence de groupe témoin dans cette étude, pour mémoire le taux national de participation pour les minorités était d'environ $54 \%$ ). Parmi les patients ayant effectué la coloscopie, deux tiers déclaraient dans un questionnaire qu'ils n'auraient pas effectue' cette coloscopie sans accompagnement.

Des résultats similaires ont également été retrouvés dans les études s'intéressant à la participation des femmes au dépistage des cancers du sein par mammographie ou au dépistage des cancers du col de l'utérus. Ainsi, un programme d'intervention a montré une amélioration de la participation des femmes au dépistage des cancers du sein dans une communauté de femmes défavorisées de l'agglomération de Denver [31]. Cette étude visait également à comparer deux types de stratégie d'accompagnement. La première stratégie consistait à organiser l'accompagnement grâce à une rencontre en face-à-face. La seconde consistait à organiser l'accompagnement à l'aide de rendez-vous téléphoniques précédés de l'envoi d'un kit d'information. Les deux méthodes d'accompagnement ont donné des résultats similaires sur la participation des femmes à la mammographie.

Une étude américaine s'intéressant au dépistage des cancers du col de l'utérus dans une communauté de femmes immigrées de Corée du Sud a montré une augmentation spectaculaire, de près de $71 \%$, de la participation à ce dépistage après l'intervention d'un accompagnateur [32]. Toutefois, il convient de relativiser l'effet de l'accompagnateur, car son action a été doublée d'une session d'information. Le bras témoin a reçu une information généraliste sur la santé incluant des notions sur le cancer du col de l'utérus, alors que la session d'information a été centrée sur le cancer du col de l'utérus dans le bras intervention.

\section{Influence des programmes d'accompagnateur}

intervenant sur des patients ayant rec uun diagnostic de cancer

À côté des études s'intéressant au dépistage des cancers, des programmes d'accompagnement ont également été envisagés sur des patients après le diagnostic. Bien que moins nombreuses, ces études rapportent des résultats intéressants.
Une étude conduite au sein du Harlem Hospital Tumor Registry mérite une attention particulière $[33,20]$. En effet, son objectif était d'évaluer le bénéfice, en termes d'amélioration du stade au diagnostic, de l'intervention pionnière lancée par le Dr Freeman en 1990. Les auteurs ont mis en évidence une amélioration du stade au diagnostic après la mise en place de l'intervention, le taux de stade de découverte tardif (stade III et IV) passé de $49 \%$ avant l'intervention à $21 \%$ dans la dernière période d'étude. De plus, la survie brute à cinq ans des patientes après l'intervention a augmenté jusqu'à 70,2 \%, contre $39 \%$ avant l'intervention. Néanmoins, en dehors des limites inhérentes au biais d'avance au diagnostic, la part attribuable àl'action de l'accompagnateur dans ce glissement des stades de découverte vers des stades plus précoces reste incertaine puisque, la mise en place d'un accompagnateur a été accompagnée par la mise en place d'une consultation gratuite et d'un programme d'éducation à la santé. Des études visant spécifiquement l'impact de l'introduction d'un programme d'accompagnement sur une amélioration du stade d'extension du cancer au moment de sa découverte mériteraient d'être conduites.

L'adhésion aux traitements adjuvants étant l'un des facteurs contribuant à la formation des inégalités sociales, des études ont également été conduites afin d'améliorer l'accès à ces traitements. Une intervention proposée au sein d'une communauté d'indiens d'Amérique du Dakota du Sud a montré une amélioration de la compliance à la radiothérapie [34]. À l'opposé, un vaste essai contrôlé randomisé portant sur 487 femmes atteintes d'un cancer du sein ou d'un cancer gynécologique n'a pas été en mesure de montrer une augmentation de la participation aux traitements adjuvants dans le groupe ayant $\mathrm{rec}_{3} \mathrm{u}$ l'accompagnement. Les auteurs soulignent que le taux d'accès aux traitements adjuvants était particulièrement élevé dans la population de l'étude en dehors de l'intervention, évoquant, de ce fait, un manque de puissance [35].

Une étude a également rapporté une amélioration du suivi grâce à une plus grande participation des patientes atteintes d'un cancer du sein aux consultations [36]. Une autre a montre' comment les accompagnateurs amélioraient le bien-être des patients pendant le traitement [37].

\section{Pertinence d'une intervention d'accompagnement au dépistage en France}

Les études visant spécifiquement les populations en situation de précarité sont rares en France. En effet, si des efforts sont régulièrement entrepris pour lutter contre les inégalités sociales, ces efforts relèvent le plus souvent d'initiative locale et sont rarement évalués. Toutefois, une étude concernant le dépistage des cancers du col utérin dans les quartiers défavorisés de Marseille mérite d'être citée [38]. Cette étude a montré qu'une lettre d'invitation des femmes en vue de la réalisation d'un frottis était insuffisante. L'ajout d'une information par des relais sociaux permettait d'augmenter la participation, qui demeurait néanmoins très faible (augmentation de la participation de 1,42 à5,58 \%). 
Les études d'intervention proposant un accompagnement à des populations socialement défavorisées ont montré leur efficacité en Amérique du Nord. Dés lors que se pose la question de leur reproductibilite' en France et plus largement en Europe, il importe de savoir si les déterminants sociaux de la santé sont comparables entre les deux continents. Aux ÉtatsUnis, l'accès à une assurance maladie est un facteur majeur dans la formation des inégalités sociales de santé [39]. Bien que l'accès à une complémentaire santé soit inégalitaire en France, cette question est probablement moins prépondérante dans notre pays. Il est à noter que les études d'accompagnement proposées aux États-Unis ne proposent généralement pas de couvrir les frais engagés. Cela nous laisse à penser que cette différence ne représente pas un obstacle majeur concernant la reproductibilite' de ce type d'intervention en France. Les autres déterminants sociaux de la santé rapportés aux États-Unis, comme la distance d'accès aux soins, le manque de soutien social et le revenu ont été également identifiés comme tels en France.

Une autre différence potentielle en termes de reproductibilité de ces actions concerne la population cible. Aux ÉtatsUnis, ces actions sont proposées à des communautés souvent ethniquement déterminées (afro-américain, etc...), pour lesquelles il existe une inégalité face à un indicateur de santé. En France, il n'est pas d'usage de favoriser la culture communautaire et l'identification des populations cible repose davantage sur l'appartenance à un mode spécifique de protection sociale ou sur le lieu de résidence. Ce dernier a le double avantage d'être facilement opérationnel et de correspondre à un vrai déterminant de la santé dans le domaine des cancers, concernant, par exemple, l'accès au dépistage [15], la détermination des filières de soins $[40,41]$ ou bien encore le pronostic [9]. Toutefois, même s'il existe des différences dans l'identification des populations précaires nécessitant un accompagnement, cela ne constitue pas une difficulte' majeure dans l'évaluation de l'intérêt du dispositif.

Une étude d'intervention multicentrique à unité de randomisation collective sera conduite prochainement en France, afin d'améliorer la participation des personnes les plus défavorisées au dépistage organisé des cancers colorectaux dans plusieurs départements du Cancéropôle Nord-Ouest. Deux groupes parallèles, stratifiés sur le type d'IRIS (plus petite entité géographique pour laquelle on dispose de statistiques socioéconomiques), seront construits :

- un groupe témoin, dans lequel les stratégies d'invitation des patients au dépistage et de remise des tests ne seront pas modifiées par rapport à l'organisation déjà en vigueur et qui correspondront aux directives du cahier des charges national ;

- un groupe intervention, dans lequel l'action d'un accompagnateur au dépistage sera ajoutée au dispositif déjà en vigueur.

En moyenne, 2500 sujets par bras (intervention/témoin) seront inclus dans chaque département.

Comme dans les études conduites aux États-Unis, le rôle de l'accompagnateur dans chaque département sera d'entrepren- dre auprès de la population qui lui aura été affectée l'ensemble des actions possibles permettant de lever les barrières empêchant ou retardant la réalisation du dépistage. Le critère de jugement principal sera le taux de participation au programme de dépistage et les délais de sa réalisation. Une évaluation économique (étude coût-efficacité) sera également conduite. Enfin, un questionnaire sera envoyé à un échantillon représentatif pour investiguer les mécanismes psychologiques de l'action des accompagnateurs.

Une étude de faisabilité est actuellement conduite dans le département de l'Orne afin de détailler les besoins spécifiques des personnes en difficulte' et d'adapter les actions de l'accompagnateur.

\section{Conclusion}

À l'image du nouveau plan cancer, les objectifs de la politique nationale de santé intègrent désormais la réduction des inégalités sociales. Les programmes d'accompagnement ont fait à l'étranger la preuve de leur efficacité, en particulier, dans l'amélioration de l'accès des populations défavorisées au dépistage ou aux traitements adjuvants des cancers. La conduite de ce type d'étude d'intervention en France représente une voie de recherche particulièrement prometteuse dans le domaine des inégalités sociales et géographiques de santé. L'évaluation économique et l'évaluation des mécanismes psychologiques de l'action des accompagnateurs est indispensable à la mise en place d'une politique globale et raisonnée de réduction des inégalités de santé.

\section{Conflit d'intérêt}

Les auteurs déclarent n'avoir aucun conflit d'intérêt.

\section{Remerciements}

Les auteurs tiennent àremercier l'ensemble du personnel de la structure de gestion du dépistage du département de l'Orne et, plus particulièrement, sa directrice Mme Annick Notari. Les auteurs souhaitent également remercier Mlle Estèle Bianeiss. Enfin, nous souhaitons remercier le Cancéropôle Nord-Ouest pour son soutien financier.

\section{Références}

[1] Leclerc A, Kaminski M, Lang T. Inégaux face à la santé. Paris: Inserm-La découverte; 2008

[2] Haut comite' de la sante' publique. La sante' en France 2002. Paris: La documentation franc aise; 2002. 410p.

[3] Chauvin P, Lebas J. Inégalités et disparités sociales de santé. In: Bourdillon F, Brücker G, Tabuteau D, editors. Traité de santé publique. $2^{\mathrm{e}}$ édition revue et augmentée, Paris: Flammarion Médecine Sciences; 2007. p. 331-41.

[4] Mackenbach JP, Kunst AE, Cavelaars A, Groenhof F, Geurts JJM, Andersen O, et al. Socioeconomic inequalities in morbidity and mortality in Western Europe. Lancet 1997;349(9066):1655-9.

[5] Woods L, Rachet B, Coleman M. Origins of socio-economic inequalities in cancer survival: a review. Ann Oncol 2006;17(1):5-19. 
[6] Hiatt R, Breen N. The social determinants of cancer: a challenge for transdisciplinary science. Am J Prev Med 2008;35(2 Suppl.):S141-50.

[7] Goldberg M, Melchior M, Leclerc A, Lert F. Epidemiology and social determinants of health inequalities. Rev Epidemiol Sante Publique 2003;51(4):381-401.

[8] Herbert C, Launoy G. Les cancers. In: Leclerc A, Fassin D, Grandjean H, et al., editors. Les inégalités sociales de santé. Paris: Inserm-La découverte; 2000

[9] Dejardin O, Bouvier A, Faivre J, Boutreux S, De Pouvourville G, Launoy G. Access to care, socioeconomic deprivation and colon cancer survival. Aliment Pharmacol Ther 2008;27(10):940-9.

[10] Dejardin O, Remontet L, Bouvier AM, Danzon A, Tretarre B, Delafosse P, et al. Socioeconomic and geographic determinants of survival of patients with digestive cancer in France. Br J Cancer 2006;95(7):944-9.

[11] Blais S, Dejardin O, Boutreux S, Launoy G. Social determinants of access to reference care centres for patients with colorectal cancer - A multilevel analysis. Eur J Cancer 2006;42(17):3041-8.

[12] Belot A, Grosclaude P, Bossard N, Jougla E, Benhamou E, Delafosse P, et al. Cancer incidence and mortality in France over the period 1980-2005. Rev Epidemiol Sante Publique 2008;56(3):159-75.

[13] Herbert C, Launoy G, Gignoux M. Factors affecting compliance with colorectal cancer screening in France: differences between intention to participate and actual participation. Eur J Cancer Prev 1997;6(1):44-52.

[14] Ioannou G, Chapko M, Dominitz J. Predictors of colorectal cancer screening participation in the United States. Am J Gastroenterol 2003; 98(9):2082-91.

[15] Pornet C, Dejardin O, Morlais F, Bouvier V, Launoy G. Socioeconomic determinants for compliance to colorectal cancer screening. A multilevel analysis. J Epidemiol Community Health 2010;64(4):318-24.

[16] Nur U, Rachet B, Parmar M, Sydes M, Cooper N, Lepage C, et al. No socioeconomic inequalities in colorectal cancer survival within a randomised clinical trial. Br J Cancer 2008;99(11):1923-8.

[17] Chaix B, Chauvin P. The contribution of multilevel models in contextual analysis in the field of social epidemiology: a review of literature. Rev Epidemiol Sante Publique 2002;50(5):489-99.

[18] Chauvin P, Parizot I, editors. Santé et expériences de soins : de l'individu à l'environnement social. Paris: Éditions Inserm-Vuibert; 2005. 300 p..

[19] Freeman H, Muth B, Kerner J. Expanding access to cancer screening and clinical follow-up among the medically underserved. Cancer Pract 1995;3(1):19-30.

[20] Freeman H. Patient navigation: a community centered approach to reducing cancer mortality. J Cancer Educ 2006;21(Suppl.):S11-4.

[21] Wells K, Battaglia T, Dudley D, Garcia R, Greene A, Calhoun E, et al. Patient navigation: state of the art or is it science? Cancer 2008;113(8): 1999-2010.

[22] Freund K, Battaglia T, Calhoun E, Dudley D, Fiscella K, Paskett E, et al. National Cancer Institute Patient Navigation Research Program: methods, protocol, and measures. Cancer 2008;113(12):3391-9.

[23] Dohan D, Schrag D. Using navigators to improve care of underserved patients: current practices and approaches. Cancer 2005;104(4):848-55.

[24] House JS. Work stress and social support. Addison-Wesley: Reading, MA; 1981.

[25] Ngoc Nguyen T, Tanjasiri S, Kagawa-Singer M, Tran J, Foo M. Community health navigators for breast- and cervical-cancer screening among cambodian and laotian women: intervention strategies and relationshipbuilding processes. Health Promot Pract 2008;9(4):356-67.
[26] Calhoun E, Whitley E, Esparza A, Ness E, Greene A, Garcia R, et al. A National Patient Navigator Training Program. Health Promot Pract 2010;11(2):205-15.

[27] Pruitt S, Shim M, Mullen P, Vernon S, Amick Br. Association of area socioeconomic status and breast, cervical, and colorectal cancer screening: a systematic review. Cancer Epidemiol Biomarkers Prev 2009;18(10): 2579-99.

[28] Nash D, Azeez S, Vlahov D, Schori M. Evaluation of an intervention to increase screening colonoscopy in an urban public hospital setting. J Urban Health 2006;83(2):231-43.

[29] Percac-Lima S, Grant R, Green A, Ashburner J, Gamba G, Oo S, et al. A culturally tailored navigator program for colorectal cancer screening in a community health center: a randomized, controlled trial. J Gen Intern Med 2009;24(2):211-7.

[30] Chen L, Santos S, Jandorf L, Christie J, Castillo A, Winkel G, et al. A program to enhance completion of screening colonoscopy among urban minorities. Clin Gastroenterol Hepatol 2008;6(4):443-50.

[31] Dignan M, Burhansstipanov L, Hariton J, Harjo L, Rattler T, Lee R, et al. A comparison of two Native American Navigator formats: face-to-face and telephone. Cancer Control 2005;12(Suppl. 2):28-33.

[32] Fang C, Ma G, Tan Y, Chi N. A multifaceted intervention to increase cervical cancer screening among underserved Korean women. Cancer Epidemiol Biomarkers Prev 2007;16(6):1298-302.

[33] Oluwole S, Ali A, Adu A, Blane B, Barlow B, Oropeza R, et al. Impact of a cancer screening program on breast cancer stage at diagnosis in a medically underserved urban community. J Am Coll Surg 2003;196(2): 180-8.

[34] Petereit D, Molloy K, Reiner M, Helbig P, Cina K, Miner R, et al. Establishing a patient navigator program to reduce cancer disparities in the American Indian communities of Western South Dakota: initial observations and results. Cancer Control 2008;15(3):254-9.

[35] Ell K, Vourlekis B, Xie B, Nedjat-Haiem F, Lee P, Muderspach L, et al. Cancer treatment adherence among low-income women with breast or gynecologic cancer: a randomized controlled trial of patient navigation. Cancer 2009;115(19):4606-15.

[36] Ell K, Padgett D, Vourlekis B, Nissly J, Pineda D, Sarabia O, et al. Abnormal mammogram follow-up: a pilot study women with low income. Cancer Pract 2002;10(3):130-8.

[37] Giese-Davis J, Bliss-Isberg C, Carson K, Star P, Donaghy J, Cordova M, et al. The effect of peer counseling on quality of life following diagnosis of breast cancer: an observational study. Psychooncology 2006;15(11): 1014-22.

[38] Piana L, Leandri F, Jacqueme B, Heid P, Corti J, Andrac-Meyer L, et al. Organized cervical cancer screening for underpriviledged women. Bull Cancer 2007;94(5):461-7.

[39] Ward E, Halpern M, Schrag N, Cokkinides V, DeSantis C, Bandi P, et al. Association of insurance with cancer care utilization and outcomes. CA Cancer J Clin 2008;58(1):9-31.

[40] Herbert C, Lefevre H, Gignoux M, Launoy G. Influence of social and occupational class and area of residence on management and survival in patients with digestive tract cancer: a population study in the Calvados area (France). Rev Epidemiol Sante Publique 2002;50(3):253-64.

[41] Madelaine J, Guizard AV, Lefevre H, Lecarpentier MM, Launoy G. Diagnosis, treatment, and prognosis of lung cancer in the Manche (France) (1997-1999) according to patients socioeconomic characteristics. Rev Epidemiol Sante Publique 2002;50(4):383-92. 